\title{
Population and Interconversion of Neutral and Zwitterionic Forms of L-Alanine in Solution
}

\author{
Young Kee Kang," Byung Jin Byun, Yong Hyun Kim, ${ }^{\dagger}$ Yun Ho Kim, ${ }^{\dagger}$ Dong Hwa Lee, and Joo Yun Lee \\ Department of Chemistry: Chungbuk National Lniversitw, Cheongin, Chungbuk 361-763, Korea \\ ${ }^{*}$ E-mail: wkangrachungbuk.ac.kr \\ ${ }^{\dagger}$ Daejeon Science High School, Daejeon 305-338, Korea \\ Recerved Hav 2, 2008
}

\begin{abstract}
The conformational study on neutral and zwitterionic L-alanines (N-Ala and Z-Ala, respectively) and the transition state (TS) for their interconversion is carried out using ab initio HF and density functional B3LYP methods with the self-consistent reaction field method in the gas phase and in solution. At both the HF and B3LYP levels of theory, the local minimum NI for N-Ala is found to be most preferred in the gas phase and a weak asy mmetric bifurcated hydrogen bond between the amino hydrogens and the carbonyl oxygen appears to play a role in stabilizing this conformation. The local minima $\mathrm{N} 2 \mathrm{a}$ and $\mathrm{N} 2 \mathrm{~b}$ are found to be the second preferred conformations. which seem to be stabilized by a hydrogen bond between the amino nitrogen and the carboxylic hydrogen. The relative stability of the local minimum $\mathrm{N} 2 \mathrm{~b}$ is remarkably increased in solution than that in the gas phase. The local minimum N2b becomes more stable than the local minimum N2a in most of the solution. On the whole the relative free energies of Z-Ala and TS become more lowered. as the solvent polarity increases. $\mathrm{N}$-Ala prevails over Z-Ala in aprotic solutions but Z-Ala is dominantly populated in ethanol and water. In aprotic solutions. the population of Z-Ala increases somew hat with the increase of solvent polarity. The barrier to Z-Ala-to-N-Ala interconversion increases on the whole with the increase of solvent polarity', which is caused by the increase of stability for Z-Ala.
\end{abstract}

Key Words : L-Alanine. Population, Interconversion. Solvent effects

\section{Introduction}

L-Alanine exists as a zwitterion in the crystal ${ }^{1.2}$ and in aqueous solution. ${ }^{3.4}$ whereas it has a neutral form in the gas phase ${ }^{5-7}$ as known for other amino acids. Because the zwitterion possesses an extremely high electric moment. it is very soluble in water but nearly insoluble in organic solvents. ${ }^{3}$ However. the neutral form has a greater affinity for organic solvents than for water. due to the relatively smaller electric moment. ${ }^{3}$ It was suggested that the concentration ratio of zwitterions to neutral molecules should decreases. as the dieletric constant (i.e. the polarity) of the solvent is lowered. ${ }^{3}$

Considerable quantum chemical computations have been carried out on the conformational preferences of neutral alanine in the gas phase. ${ }^{8-15}$ and neutral and/or zwitterionic alanines in aqueous solution ${ }^{13.1+16 ;=1}$ and in a $\mathrm{KBr}$ matrix $=2.23$ For most of the previous works in solution. solvents were considered as a continuum and the self-consistent reaction field (SCRF) methods were employed to calculate solvation free energies. ${ }^{13.14 .16 .22 .23}$ A few works have adopted the models of zwitterionic ${ }^{17-36}$ or neutral ${ }^{30}$ alanine with explicit waters embedded in a SCRF.

In the gas phase the most preferred conformation and up to 13 local minima for the neutral alanine were identified depending on the levels of theory and starting structures. In particular. the zwitterionic alanine can be located as a local minimum at the $\mathrm{HF} / 6-31 \mathrm{G}(\mathrm{d})$ level of theory but it is not a local minimum at $\mathrm{B} 3 \mathrm{LYP} / 6-3 \mathrm{I}+\mathrm{G}(\mathrm{d})$ and $\mathrm{B} 3 \mathrm{LYP} / 6-$
$311++G(d . p)$ levels of theory ${ }^{13}$ It was suggested that at least two water molecules are needed to form a stable alanine zwitterion-water cluster in the gas phase. ${ }^{20}$ The zwitterionic form was found to be more stable than the neutral form when the solvation shell reaches eight water molecules. ${ }^{21}$

In water. the zwitterionic alanine was found to be more stable by about $3-4 \mathrm{kcal} / \mathrm{mol}$ in free energy than the neutral alanine using polarizable continuum models. ${ }^{16.2}$ There are limited works reported on the interconversion between neutral and zwitterionic alanines in water ${ }^{20.24}$ and in organic solvents. ${ }^{25}$ in which only representative conformations of the neutral alanine were employed.

We studied here all feasible conformations for neutral and zwitterionic L-alanines and the transition states for their interconversion at the HF and B3LYP levels of theory with the conductor-like polarizable continuum model (CPCM) in solution in order to investigate their relative populations and interconversion with the increase of solvent polarity.

\section{Computational Methods}

Chemical structure and atomic labels of L-alanine are shown in Figure 1. All $a b$ initio HF and hybrid density functional B3LYP calculations were carried out using the Gaussian 03 package. ${ }^{26}$ The 13 local minima of $L-$ and $D-$ alanines optimized at the HF/6-311++G(d.p) level of theory ${ }^{10}$ and the 9 local minima of Ac-L-Ala-NHMe optimized by the ECEPP $/ 2$ force field ${ }^{27}$ were used as starting conformations for optimization of the neutral L-alanine (N-Ala) at $\mathrm{HF} / 6-$ 


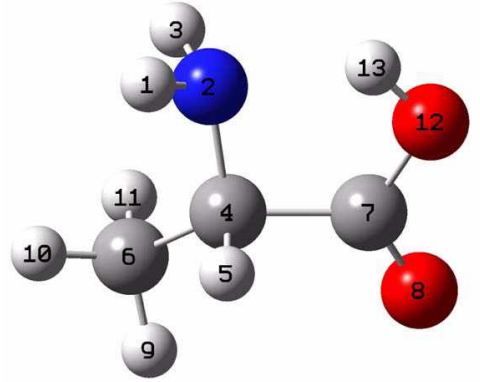

Figure 1. Chemical structure and atomic labels for L-alanine

$31+G(d)$ and B3LYP $/ 6-31+G(d)$ levels of theory in the gas phase. The local minima at these HF and B3LYP levels of theory were edited by the GaussView ${ }^{28}$ to obtain the starting structures for optimization of the zwitterionic L-alanine (ZAla) in the gas phase. After optimization. a single local minimum for Z-Ala was located at the HF/6-31+G(d) level of theory. but no stationary point was found at the B3LYP/6$3 \mathrm{I}+\mathrm{G}(\mathrm{d})$ level of theory, as seen previously. ${ }^{13}$ The transition state (TS) between $\mathrm{N}$-Ala and Z-Ala was located using the Synchronous Transit and Quasi-Newton (STQN) method ${ }^{29}$ with the QST3 option of the Gaussian $03^{3(1}$ at the HF/6$3 \mathrm{I}+\mathrm{G}(\mathrm{d})$ level of theory. $\mathrm{N}$-Ala with a similar configuration of amino and carboxylic groups to Z-Ala was chosen as initial structure in generating the TS structure.

We employed the conductor-like polarizable continuum model (CPCM) ${ }^{31, \hat{3} 2}$ intelemented in the Gaussian 03 package. ${ }^{26}$ to compute solvation free energies $\left(\Delta G_{\text {solv }}\right)$ at the $\mathrm{HF} /$ $6-31+G(d)$ level of theory with the united atom Koln-Sham (UAKS) cavities, which are the united atom topological model (UATM) radii optimized at the density functional $\mathrm{PBE} 0 / 6-3 \mathrm{IG}(\mathrm{d})$ level of theory. ${ }^{33.34}$ The solvation free energy is the sum of the electrostatic free energy and the nonelectrostatic energy terms. ${ }^{35}$ The latter is composed of the cavitation, dispersion, and repulsion energy terms. For CPCM-UAKS calculations. the default average areas of 0.2 $\mathrm{A}^{2}$ for tesserae were used. The solvents considered here are chloroform. dichloroethane, ethanol acetonitrile. DMSO. and water. whose dielectric constants are 4.9, 10.4. 24.6. 36.6. 46.7 and 78.4 at $25^{\circ} \mathrm{C}$, respectively. ${ }^{31}$ Recently. the CPCM-UAKS calculations for a number of neutral and charged organic molecules at the $\mathrm{HF} / 6-31+\mathrm{G}(\mathrm{d}) / \mathrm{HF} / 6-$ $31+\mathrm{G}(\mathrm{d})$ and $\mathrm{HF} / 6-31+\mathrm{G}(\mathrm{d}) / \mathrm{B} 3 \mathrm{LYP} / 6-31+\mathrm{G}(\mathrm{d})$ levels of theory provided hydration free energies in agreement with available experimental data. ${ }^{36}$

All local minima for N-Ala optimized at $\mathrm{HF} / 6-3 \mathrm{I}+\mathrm{G}$ (d) and $\mathrm{B} 3 \mathrm{LYP} / 6-3 \mathrm{I}+\mathrm{G}(\mathrm{d})$ levels of theory in the gas phase were used as starting structures for optimizations at $\mathrm{CPCM}$ $\mathrm{HF} / 6-3 \mathrm{l}+\mathrm{G}(\mathrm{d})$ and CPCM B3LYP/6-31+G(d) levels of theory in solution. respectively. Z-Ala and TS optimized at the $\mathrm{HF} / 6-31+\mathrm{G}(\mathrm{d})$ level of theory in the gas phase were used as starting structures for optimizations at both the CPCM $\mathrm{HF} / 6-31+\mathrm{G}(\mathrm{d})$ and CPCM B3LYP/6-31+G(d) levels of theory in solution. The B3LYP/6-311++G(d.p) single-point energies were calculated for all local minima and transition states of $L$-alanine located at $C P C M H F / 6-3 I+G(d)$ and
CPCM B3LYP/6-31+G(d) levels of theory in solution.

Vibrational frequencies were calculated for all stationary points at both the HF and B3LYP levels of theory in the gas phase and in solution, which were used to compute enthalpies and Gibbs free energies with the scale factors of 0.89 and 0.98 at HF and B3LYP levels of theory, respectively, at $25^{\circ} \mathrm{C}$ and $\mathrm{I} \mathrm{atm}$. These scale factors were chosen to reproduce experimental frequencies for the amide I band of $\mathrm{N}$ methylacetamide in Ar and $\mathrm{N}_{2}$ matrixes. ${ }^{37}$ The zero-point energy correction and the thermal energy corrections were used to calculate the enthalpy $(H)$ and entropy $(S)$ of each confommation ${ }^{20,38}$ The analysis uses the standard thermodynamic expressions for an ideal gas in the canonical ensemble. Each transition state was confirmed by checking whether it had one imaginary frequency after frequency calculations at both the HF and B3LYP levels of theory. The relative total free energy $(\Delta G)$ for each conformation in solution was computed by taking the sum of the relative conformational free energy $\left(\Delta E_{c}\right)$, the thermal contributions, and the entropic contribution. The relative conformational free energy $\left(\Delta E_{v}\right)$ is the sum of the conformational electronic energy $\left(\Delta E_{r . s}\right)$ and the relative solvation free energy $\left(\Delta \Delta G_{\text {solv }}\right)$ in solution. The relative total free energies are used here to interpret the population and interconversion between $\mathrm{N}$-Ala and Z-Ala in the gas phase and in solution.

\section{Results and Discussion}

Conformational Preferences in the Gas Phase. Thermodynamic properties of N-Ala. Z-Ala, and their TS calculated at $\mathrm{HF} / 6-3 \mathrm{l}+\mathrm{G}(\mathrm{d}), \mathrm{B} 3 \mathrm{LYP} / 6-3 \mathrm{ll}++\mathrm{G}(\mathrm{d} . \mathrm{p}) / \mathrm{HF} / 6-3 \mathrm{l}+\mathrm{G}(\mathrm{d})$, $\mathrm{B} 3 \mathrm{LYP} / 6-3 \mathrm{l}+\mathrm{G}(\mathrm{d})$. and $\mathrm{B} 3 \mathrm{LYP} / 6-31 \mathrm{l}+\mathrm{G}(\mathrm{d} . \mathrm{p}) / \mathrm{B} 3 \mathrm{LYP} / 6-$ $31+\mathrm{G}(\mathrm{d})$ levels of theory in the gas phase are listed in Tables 1-4. respectively. The conformations of all feasible local minima and TS found at $\mathrm{HF} / 6-3 \mathrm{l}+\mathrm{G}(\mathrm{d})$ and $\mathrm{B} 3 \mathrm{LYP} / 6-$ $31+\mathrm{G}(\mathrm{d})$ levels of theory in the gas phase are shown in Figure 2. Their selected bond lengths, bond angles, and torsion angles optimized at $\mathrm{HF} / 6-31+\mathrm{G}(\mathrm{d})$ and $\mathrm{B} 3 \mathrm{LYP} / 6-$ $31+\mathrm{G}$ (d) levels of theory in the gas phase are listed in the Supporting Information.

At the $\mathrm{HF} / 6-31+\mathrm{G}(\mathrm{d})$ level of theory. the 12 local minima are found for N-Ala in the gas phase (Tables 1 and 2). The eight local minima of them such as N1. N2b. N4, N5, N8. N9. N10. and N11 correspond to the local minima I. IIB. IIIB. IVA. VB. VI. VII, and VIIA for L-alanine found at the $H F / 6-311++G(d, p)$ level of theory. respectively. which are listed in Tables 2 and 3 of ref 10 . The local minimum N2a is also identified as a local minimum at MP2/6-311++G(d.p $)^{7}$ and B3LYP/aug-cc-pVDZ ${ }^{11}$ levels of theory. The conformations of the remaining three local minima such as N3. N6, and $\mathrm{N} 7$ are similar to those of the local minima N8. N1. and $\mathrm{N} 4$, respectively. except having the different orientations of amide hydrogens. The relative conformational stabilities of $\mathrm{N}$-Ala in the gas phase are calculated to be in the order $\mathrm{Nl}>$ $\mathrm{N} 3>\mathrm{N} 4>\mathrm{N} 5>\mathrm{N} 6>\mathrm{N} 7>\mathrm{N} 8>\mathrm{N} 2 \mathrm{a} \approx \mathrm{N} 2 \mathrm{~b}>\mathrm{N} 9>\mathrm{N} 10>$ $\mathrm{N} 11$ at the $\mathrm{HF} / 6-31+\mathrm{G}(\mathrm{d})$ level of theory by both the relative electronic energies $\left(\Delta E_{\mathrm{e}}\right)$ and free energies $(\Delta G)$. At the 
Table 1. Themodynamic Properties of Neutral and Zwitterionic L-Alanines and Their Transition State at the HF/6-31+G(d) Level of Theory in the Gas Phase and in Solution ${ }^{2}$

\begin{tabular}{|c|c|c|c|c|c|c|c|c|c|c|c|c|c|c|c|}
\hline solvent & & $\mathrm{Nl}$ & $\mathrm{N} 2 \mathrm{a}$ & $\mathrm{N} 2 \mathrm{~b}$ & $N 3$ & $\mathrm{~N} 4$ & N5 & N6 & N7 & N8 & N9 & $\mathrm{NlO}$ & $\mathrm{Nll}$ & $Z$ & $\mathrm{TS}$ \\
\hline \multirow[t]{3}{*}{ gas phase } & $\Delta E_{\mathrm{e}}$ & 0.00 & 2.38 & 2.47 & 1.22 & 1.56 & 1.69 & 1.80 & 1.92 & 2.25 & 7.14 & 8.48 & 8.86 & 24.66 & 25.37 \\
\hline & $\Delta H$ & 0.00 & 2.54 & 2.58 & 1.22 & 1.52 & 1.61 & 1.68 & 1.80 & 2.16 & $7 .(12$ & 8.17 & 8.66 & 24.63 & 23.09 \\
\hline & $\Delta G$ & 0.00 & 2.96 & 2.99 & 1.08 & 1.28 & 1.67 & 1.83 & 1.97 & 2.23 & 7.01 & 8.25 & 8.53 & 24.82 & 24.17 \\
\hline \multirow[t]{3}{*}{$\mathrm{CHCl}_{3}$} & $\Delta E_{\mathrm{e}}$ & 0.00 & 0.59 & 0.52 & 0.99 & 1.41 & 1.30 & 1.23 & 1.47 & 1.60 & 4.69 & 5.92 & 5.91 & 7.76 & 16.94 \\
\hline & $\Delta H$ & 0.00 & 0.82 & 0.67 & 1.00 & 1.38 & 1.26 & 1.15 & 1.40 & 1.56 & 4.66 & 5.75 & 5.85 & 8.75 & 14.63 \\
\hline & $\Delta G$ & 0.00 & 1.24 & 1.06 & 0.85 & 0.96 & 1.31 & 1.22 & 1.44 & 1.51 & 4.71 & 5.79 & 5.70 & 7.86 & 15.83 \\
\hline \multirow[t]{3}{*}{$\mathrm{CH}_{2} \mathrm{ClCH}_{2} \mathrm{Cl}$} & $\Delta E_{\mathrm{e}}$ & 0.00 & 0.25 & 0.15 & 0.95 & 1.38 & 1.19 & 1.12 & 1.38 & 1.48 & 4.25 & 5.46 & 5.34 & 4.61 & 15.64 \\
\hline & $\Delta H$ & 0.00 & 0.49 & 0.30 & 0.96 & 1.36 & 1.16 & 1.04 & 1.32 & 1.44 & 4.24 & 5.31 & 5.29 & 5.65 & 13.34 \\
\hline & $\Delta G$ & 0.00 & 0.91 & 0.70 & 0.82 & 0.92 & 1.16 & 1.08 & 1.35 & 1.39 & 4.30 & 5.34 & 5.15 & 5.51 & 14.55 \\
\hline \multirow[t]{3}{*}{$\mathrm{EtOH}$} & $\Delta E_{\mathrm{e}}$ & 000 & 1.38 & 0.93 & 1.00 & 1.28 & 1.14 & 0.96 & 1.45 & 1.47 & 2.98 & 4.89 & 4.00 & -3.14 & 15.40 \\
\hline & $\Delta H$ & 0.00 & 1.77 & 1.01 & 1.08 & 1.20 & 1.16 & 0.81 & 1.32 & 1.50 & 3.03 & 4.92 & 4.06 & -1.48 & 13.74 \\
\hline & $\Delta G$ & 0.05 & 2.08 & 1.41 & 0.99 & 0.00 & 1.12 & 0.93 & 1.33 & 1.37 & 3.27 & 500 & 4.06 & -1.18 & 15.06 \\
\hline \multirow[t]{3}{*}{$\mathrm{CH}_{3} \mathrm{CN}$} & $\Delta E_{\mathrm{e}}$ & 0.10 & 0.09 & 0.00 & 1.01 & 1.48 & 1.21 & 1.14 & 1.43 & 1.50 & 4.012 & 5.21 & 5.01 & 2.59 & 14.91 \\
\hline & $\Delta H$ & 0.00 & 0.23 & 0.05 & 0.92 & 1.35 & 1.08 & 0.96 & 1.27 & 1.37 & 3.90 & 4.96 & 4.86 & 3.55 & 12.51 \\
\hline & $\Delta G$ & 000 & 0.55 & 0.42 & 0.76 & 0.90 & 1.08 & 1.00 & 1.27 & 1.30 & 3.96 & 4.99 & 4.76 & 3.47 & 13.72 \\
\hline \multirow[t]{3}{*}{ DMSO } & $\Delta E_{\mathrm{e}}$ & 0.15 & 0.12 & 0.00 & 1.07 & 1.50 & 1.26 & 1.15 & 1.45 & 1.55 & 4.09 & 5.27 & 5.08 & 2.39 & 14.82 \\
\hline & $\Delta H$ & 0.01 & 0.21 & 0.00 & 0.93 & 1.32 & 1.08 & 0.93 & 1.23 & 1.37 & 3.93 & 4.98 & 4.89 & 3.30 & 12.38 \\
\hline & $\Delta G$ & 0.00 & 0.57 & 0.38 & 0.75 & 0.79 & 0.99 & 0.96 & 1.22 & 1.28 & 3.98 & 5.01 & 4.74 & 3.21 & 13.58 \\
\hline \multirow[t]{3}{*}{ water } & $\Delta E_{\mathrm{e}}$ & 0.00 & 1.26 & 0.67 & 0.98 & 1.18 & 0.95 & 0.83 & 1.39 & 1.47 & 2.62 & 4.30 & 3.51 & -4.63 & 14.94 \\
\hline & $\Delta H$ & 0.00 & 1.66 & 0.73 & 1.09 & 1.10 & 0.93 & 0.68 & 1.28 & 1.52 & 2.65 & 3.71 & 3.51 & -2.92 & 13.34 \\
\hline & $\Delta G$ & 0.00 & 2.05 & 1.18 & 1.04 & 0.33 & 0.79 & 0.81 & 1.30 & 1.41 & 3.03 & 5.42 & 3.62 & -2.60 & 14.67 \\
\hline
\end{tabular}

" $\Delta E_{\mathrm{e}}, \Delta H$, and $\Delta G$ are relative electronic energies, enthalpies, and Gibbs free energies, respectively", units in kcal mol. $\Delta H$ and $\Delta G$ are calculated at 25

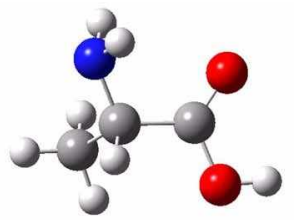

N1

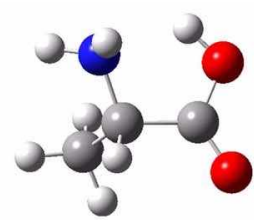

$\mathrm{N} 2 \mathrm{a}$

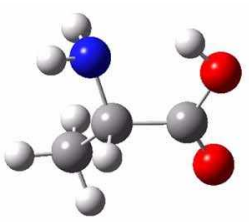

$\mathrm{N} 2 \mathrm{~b}$

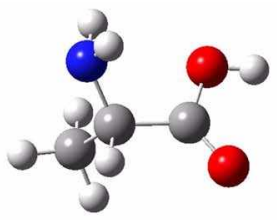

N3

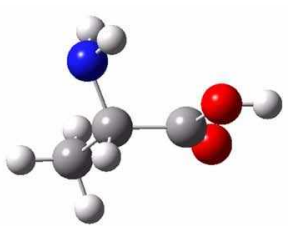

N4

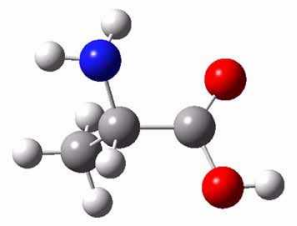

N5

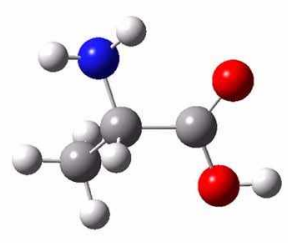

N6

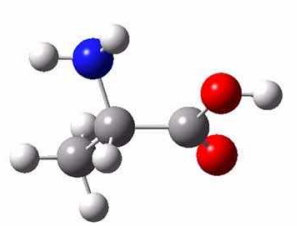

N7

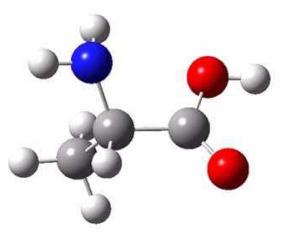

N8

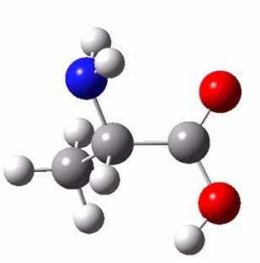

N9

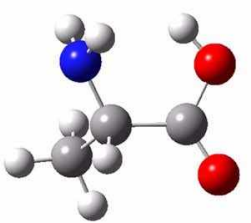

N10

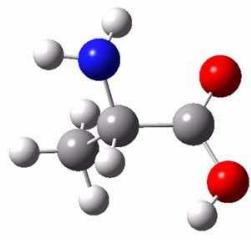

N11

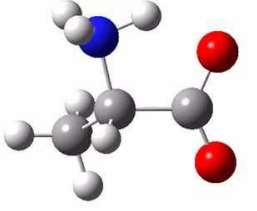

Z

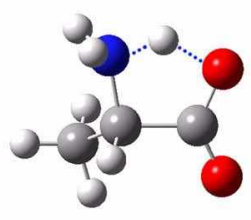

TS

Figure 2. Conformations of all feasible local minina for N-Ala and Z-Ala and TS found at HF/6-31+G(d) and B3LYP/6-31+G(d) levels of theory in the gas phase. Confonmations Nl-N1l are for N-Ala. The local minimum of Z-Ala is denoted by $Z$.

B3LYP/6-31l++G(d.p) $/ / \mathrm{HF} / 6-3 \mathrm{l}+\mathrm{G}$ (d) level of theory, the two local minima $\mathrm{N} 2 a$ and $\mathrm{N} 2 b$, however. become more farored than the local minimum $\mathrm{N} 3$ but the relative stabilities of the other local minima are the same as those at the
$\mathrm{HF} / 6-31+\mathrm{G}(\mathrm{d})$ level of theory. The calculated stabilities of local minima at HF/6-31+G(d) and B3LYP/6-311++G(d.p) $/ /$ $\mathrm{HF} / 6-31+\mathrm{G}(\mathrm{d})$ levels of theory are similar to those by the $\Delta E_{\mathrm{e}}$ at $\mathrm{HF} / 6-3 \mathrm{ll}+\mathrm{G}(\mathrm{d} . \mathrm{p})$ and $\mathrm{B} 3 \mathrm{LYP} / 6-31 \mathrm{l}+\mathrm{G}(\mathrm{d}, \mathrm{p})$ levels 
Table 2. Thennodynanic Properties of Nentral and Zwitterionic L-Alanines and Their Transition State at the B3LYP/6-311++G(d,p)//HF/6$31+\mathrm{G}(\mathrm{d})$ Level of Theory in the Gas Phase and in Solution ${ }^{\prime \prime}$

\begin{tabular}{|c|c|c|c|c|c|c|c|c|c|c|c|c|c|c|c|}
\hline solvent & & $\mathrm{Nl}$ & $\mathrm{N} 2 \mathrm{a}$ & $\mathrm{N} 2 \mathrm{~b}$ & N3 & $\mathrm{N} 4$ & N5 & N6 & N7 & N8 & N9 & $\mathrm{NlO}$ & N1l & 2 & TS \\
\hline \multirow[t]{3}{*}{ gas phase } & $\Delta E_{\varepsilon}$ & 000 & 0.36 & 0.40 & 1.10 & 1.34 & 1.35 & 1.48 & 2.01 & 2.29 & 5.74 & 608 & 7.01 & 17.55 & 14.58 \\
\hline & $\Delta H$ & 000 & 0.52 & 0.51 & 1.11 & 1.30 & 1.27 & 1.35 & 1.90 & 2.20 & 5.62 & 5.78 & 6.80 & 17.52 & 12.29 \\
\hline & $\Delta G$ & 000 & 0.95 & 0.92 & 0.96 & 1.06 & 1.33 & 1.51 & 2.07 & 2.27 & 5.61 & 5.85 & 6.68 & 17.71 & 13.37 \\
\hline \multirow[t]{3}{*}{$\mathrm{CHCl}_{3}$} & $\Delta E_{\mathrm{e}}$ & 1.77 & 0.21 & 0.00 & 2.67 & 3.00 & 2.78 & 2.74 & 3.36 & 3.43 & 4.96 & 5.34 & 5.89 & 6.14 & 6.75 \\
\hline & $\Delta H$ & 1.62 & 0.30 & 0.00 & 2.54 & 2.82 & 2.60 & 2.50 & 3.14 & 3.24 & 4.78 & 5.03 & 5.68 & 6.98 & 4.30 \\
\hline & $\Delta G$ & 1.24 & 0.33 & 0.00 & 1.99 & 2.02 & 2.26 & 2.18 & 2.79 & 2.80 & 4.44 & 4.69 & 5.14 & 5.70 & 5.11 \\
\hline \multirow[t]{3}{*}{$\mathrm{CH}_{2} \mathrm{ClCH}_{2} \mathrm{Cl}$} & $\Delta E_{\mathrm{e}}$ & 2.18 & 0.25 & 0.00 & 3.04 & 3.38 & 3.08 & 3.03 & 3.67 & 3.70 & 4.90 & 5.29 & 5.73 & 3.48 & 5.80 \\
\hline & $\Delta H$ & 2.03 & 0.34 & 0.00 & 2.91 & 3.21 & 2.90 & 2.80 & 3.46 & 3.51 & 4.74 & 4.99 & 5.53 & 4.37 & 3.36 \\
\hline & $\Delta G$ & 1.63 & 0.36 & 0.00 & 2.36 & 2.37 & 2.50 & 2.45 & 3.09 & 3.06 & 4.39 & 4.63 & 4.98 & 3.83 & 4.17 \\
\hline \multirow[t]{3}{*}{$\mathrm{EtOH}$} & $\Delta E_{\varepsilon}$ & 1.14 & 0.55 & 0.00 & 2.06 & 2.28 & 1.99 & 1.76 & 2.61 & 2.67 & 2.49 & 3.76 & 3.29 & -5.35 & 4.79 \\
\hline & $\Delta H$ & 1.06 & 0.86 & 0.00 & 2.06 & 2.12 & 1.94 & 1.54 & 2.40 & 2.62 & 2.46 & 3.72 & 3.26 & -3.77 & 3.06 \\
\hline & $\Delta G$ & 0.71 & 0.77 & 0.00 & 1.57 & 0.52 & 1.50 & 1.26 & 2.01 & 2.09 & 2.30 & 3.40 & 2.87 & -3.87 & 3.97 \\
\hline \multirow[t]{3}{*}{$\mathrm{CH}_{3} \mathrm{CN}$} & $\Delta E_{\varepsilon}$ & 2.47 & 0.17 & 0.00 & 3.30 & 3.64 & 3.29 & 3.24 & 3.91 & 3.95 & 4.82 & 5.23 & 5.57 & 1.62 & 5.23 \\
\hline & $\Delta H$ & 2.32 & 0.26 & 000 & 3.17 & 3.47 & 3.11 & 3.02 & 3.70 & 3.77 & 4.66 & 4.94 & 5.37 & 2.53 & 2.79 \\
\hline & $\Delta G$ & 1.95 & 0.21 & 0.00 & 2.62 & 2.65 & 2.74 & 2.68 & 3.33 & 3.33 & 4.35 & 4.59 & 4.90 & 2.07 & 3.63 \\
\hline \multirow[t]{3}{*}{ DMSO } & $\Delta E_{\mathrm{e}}$ & 2.51 & 0.22 & 0.00 & 3.35 & 3.70 & 3.34 & 3.25 & 3.92 & 3.99 & 4.89 & 5.29 & 5.63 & 1.36 & 5.14 \\
\hline & $\Delta H$ & 2.36 & 0.31 & 0.00 & 3.22 & 3.51 & 3.16 & 3.02 & 3.70 & 3.81 & 4.73 & 5.00 & 5.44 & 2.27 & 2.70 \\
\hline & $\Delta G$ & 1.97 & 0.29 & 0.00 & 2.65 & 2.61 & 2.68 & 2.67 & 3.31 & 3.34 & 4.40 & 4.65 & 4.91 & 1.79 & 3.52 \\
\hline \multirow[t]{3}{*}{ water } & $\Delta E_{\mathrm{e}}$ & 1.42 & 0.68 & 0.00 & 2.34 & 2.49 & 2.07 & 1.92 & 2.85 & 2.97 & 2.41 & 3.43 & 3.04 & -6.60 & 4.66 \\
\hline & $\Delta H$ & 1.36 & 1.03 & 000 & 2.39 & 2.34 & 1.99 & 1.72 & 2.68 & 2.97 & 2.38 & 2.78 & 2.99 & -4.95 & 300 \\
\hline & $\Delta G$ & 0.91 & 0.96 & 0.00 & 1.88 & 1.12 & 1.40 & 1.38 & 2.25 & 2.39 & 2.31 & 4.03 & 2.64 & -5.08 & 3.87 \\
\hline
\end{tabular}

"See tootnote $a$ of Table 1 .

Table 3. Thernodynanic Properties of Neutral and Zwitterionic L-Alanines and Their Transition State at the B3LYP/6-31+G(d) Level of Theory in the Gas Phase and in Solution ${ }^{a}$

\begin{tabular}{|c|c|c|c|c|c|c|c|c|c|c|c|c|c|}
\hline solvent & & $\mathrm{Nl}$ & $\mathrm{N} 2 \mathrm{a}$ & $\mathrm{N} 2 \mathrm{~b}$ & $\mathrm{~N} 3$ & N5 & N6 & N7 & N8 & N9 & $\mathrm{Nll}$ & 2 & TS \\
\hline \multirow[t]{3}{*}{ gas phase } & $\Delta E_{\mathrm{e}}$ & 0.00 & 0.01 & 0.06 & 1.09 & 1.44 & 1.66 & 2.13 & 2.33 & 5.98 & 7.37 & & \\
\hline & $\Delta H$ & 000 & 0.13 & 0.17 & 1.12 & 1.36 & 1.49 & 1.97 & 2.23 & 5.80 & 7.09 & & \\
\hline & $\Delta G$ & 000 & 0.63 & 0.69 & 0.96 & 1.46 & 1.71 & 2.11 & 2.32 & 5.72 & 7.10 & & \\
\hline \multirow[t]{3}{*}{$\mathrm{CHCl}_{3}$} & $\overline{\Delta E_{\mathrm{e}}}$ & 1.95 & 0.08 & 0.00 & 2.83 & 3.08 & 3.11 & 3.63 & 3.70 & 5.78 & 6.88 & 4.14 & 7.38 \\
\hline & $\Delta H$ & 1.82 & 0.06 & 0.00 & 2.74 & 2.91 & 2.85 & 3.38 & 3.52 & 5.60 & 6.65 & 4.46 & 5.11 \\
\hline & $\Delta G$ & 1.47 & 0.00 & 0.08 & 2.22 & 2.58 & 2.70 & 2.99 & 3.10 & 5.30 & 6.20 & 4.67 & 5.99 \\
\hline \multirow[t]{3}{*}{$\mathrm{CH}_{2} \mathrm{ClCH}_{2} \mathrm{Cl}$} & $\Delta E_{\mathrm{e}}$ & 2.36 & 0.12 & 0.00 & 3.19 & 3.40 & 3.42 & 3.95 & 3.99 & 5.79 & 6.80 & 2.16 & 6.55 \\
\hline & $\Delta H$ & 2.24 & 0.11 & 0.00 & 3.11 & 3.24 & 3.17 & 3.71 & 3.81 & 5.63 & 6.59 & 2.66 & 4.27 \\
\hline & $\Delta G$ & 1.80 & 0.21 & 0.00 & 2.53 & 2.85 & 2.85 & 3.21 & 3.30 & 5.27 & 6.08 & 2.71 & 5.09 \\
\hline \multirow[t]{3}{*}{$\mathrm{EtOH}$} & $\Delta E_{\mathrm{e}}$ & 1.44 & 0.28 & 0.00 & 2.10 & 2.32 & 2.16 & 2.81 & 2.84 & 3.57 & 4.62 & -3.37 & 5.98 \\
\hline & $\Delta H$ & 1.60 & 0.00 & 0.20 & 2.26 & 2.42 & 1.87 & 2.53 & 2.92 & 3.65 & 4.72 & -1.32 & 4.77 \\
\hline & $\Delta G$ & 0.71 & 100 & 0.00 & 1.21 & 1.31 & 1.16 & 1.71 & 1.90 & 3.02 & 3.92 & -2.19 & 5.24 \\
\hline \multirow[t]{3}{*}{$\mathrm{CH}_{3} \mathrm{CN}$} & $\overline{\Delta E_{\mathrm{e}}}$ & 2.64 & & 0.00 & 3.4 & 3.61 & 3.64 & 4.18 & 4.19 & 5.75 & 6.69 & 0.82 & 6.06 \\
\hline & $\Delta H$ & 2.52 & & 0.00 & 3.36 & 3.47 & 3.40 & 3.95 & 4.03 & 5.61 & 6.50 & 1.43 & 3.78 \\
\hline & $\Delta G$ & 2.06 & & 0.00 & 2.78 & 3.05 & 3.05 & 3.41 & 3.49 & 5.23 & 6.02 & 1.39 & 4.61 \\
\hline \multirow[t]{3}{*}{ DMSO } & $\overline{\Delta E_{\mathrm{e}}}$ & 2.70 & 0.08 & 0.00 & 3.51 & 3.66 & 3.66 & 4.20 & 4.24 & 5.84 & 6.77 & 0.62 & 5.97 \\
\hline & $\Delta H$ & 2.59 & 0.04 & 0.00 & 3.43 & 3.53 & 3.42 & 3.97 & 4.08 & 5.70 & 6.58 & 1.23 & 3.69 \\
\hline & $\Delta G$ & 2.39 & 0.00 & 0.27 & 3.09 & 3.48 & 3.35 & 3.70 & 3.80 & 5.59 & 6.34 & 1.46 & 4.78 \\
\hline \multirow[t]{3}{*}{ Water } & $\overline{\Delta E_{\mathrm{e}}}$ & 1.47 & & 0.00 & 2.36 & 2.40 & 2.25 & 2.96 & 3.03 & 3.48 & 4.39 & -4.52 & 5.83 \\
\hline & $\Delta H$ & 1.52 & & 0.00 & 2.56 & 2.45 & 1.91 & 2.70 & 3.12 & 3.56 & 4.42 & -2.40 & 4.71 \\
\hline & $\Delta G$ & 0.71 & & 0.00 & 1.89 & 2.03 & 1.49 & 2.17 & 2.43 & 3.24 & 4.03 & -3.03 & 5.47 \\
\hline
\end{tabular}

"See footnote $a$ of Table 1 . 
Table 4. Themmodynamic Properties of Neutral and Zwitterionic L-Alanines and Their Transition State at the B3LYP/6-311++G(d.p)/ B3LYP/6-31+G(d) Level of Theory in the Gas Phase and in Solution ${ }^{\circ}$

\begin{tabular}{|c|c|c|c|c|c|c|c|c|c|c|c|c|c|}
\hline solvent & $\mathrm{Nl}$ & $\mathrm{N} 2 \mathrm{a}$ & $\mathrm{N} 2 \mathrm{~b}$ & N3 & N5 & N6 & N7 & N8 & N9 & $\mathrm{Nll}$ & 2 & TS & \\
\hline \multirow[t]{3}{*}{ gas phase } & $\Delta E_{\mathrm{e}}$ & 000 & 0.03 & 0.02 & 1.07 & 1.32 & 1.46 & 1.98 & 2.25 & 5.73 & 6.95 & & \\
\hline & $\Delta H$ & 000 & 0.15 & 0.12 & 1.10 & 1.24 & 1.29 & 1.82 & 2.15 & 5.55 & 6.68 & & \\
\hline & $\Delta G$ & 0.00 & 0.66 & 0.64 & 0.94 & 1.33 & 1.51 & 1.95 & 2.23 & 5.47 & 6.69 & & \\
\hline \multirow[t]{3}{*}{$\mathrm{CHCl}_{3}$} & $\Delta E_{\mathrm{e}}^{\prime}$ & 1.96 & 0.13 & 0.00 & 2.83 & 3.01 & 2.95 & 3.51 & 3.66 & 5.56 & 6.59 & 6.18 & 8.24 \\
\hline & $\Delta H$ & 1.83 & 0.11 & 0.00 & 2.74 & 2.84 & 2.69 & 3.27 & 3.47 & 5.38 & 6.35 & 6.49 & 5.97 \\
\hline & $\Delta G$ & 1.42 & 0.00 & 0.02 & 2.17 & 2.45 & 2.49 & 2.81 & 3.01 & 5.02 & 5.85 & 6.65 & 6.80 \\
\hline \multirow[t]{3}{*}{$\mathrm{CH}_{2} \mathrm{ClCH}_{2} \mathrm{Cl}$} & $\Delta E_{\mathrm{e}}$ & 2.36 & 0.18 & 0.00 & 3.19 & 3.32 & 3.26 & 3.82 & 3.94 & 5.56 & 6.51 & 4.28 & 7.32 \\
\hline & $\Delta H$ & 2.24 & 0.17 & 0.00 & 3.11 & 3.16 & 3.01 & 3.59 & 3.76 & 5.40 & 6.30 & 4.78 & 5.04 \\
\hline & $\Delta G$ & 1.80 & 0.27 & 0.00 & 2.53 & 2.77 & 2.69 & 3.09 & 3.25 & 5.04 & 5.79 & 4.83 & 5.86 \\
\hline \multirow[t]{3}{*}{$\mathrm{EtOH}$} & $\Delta E_{\mathrm{e}}$ & 1.47 & 0.31 & 000 & 2.15 & 2.26 & 2.06 & 2.74 & 2.85 & 3.38 & 4.37 & -0.97 & 6.42 \\
\hline & $\Delta H$ & 1.60 & 0.00 & 0.17 & 2.28 & 2.33 & 1.73 & 2.43 & 2.89 & 3.43 & 4.44 & 1.05 & 5.18 \\
\hline & $\Delta G$ & 0.74 & 1.03 & 0.00 & 1.26 & 1.25 & 1.05 & 1.65 & 1.91 & 2.83 & 3.68 & 0.22 & 5.68 \\
\hline \multirow[t]{3}{*}{$\mathrm{CH}_{3} \mathrm{CN}$} & $\Delta E_{\mathrm{e}}^{\prime}$ & 2.63 & & 0.00 & 3.43 & 3.53 & 3.47 & 4.05 & 4.14 & 5.52 & 6.40 & 2.98 & 6.77 \\
\hline & $\Delta H$ & 2.51 & & 0.00 & 3.36 & 3.39 & 3.23 & 3.82 & 3.98 & 5.38 & 6.21 & 3.59 & 4.49 \\
\hline & $\Delta G$ & 2.05 & & 0.00 & 2.78 & 2.97 & 2.88 & 3.28 & 3.43 & 5.00 & 5.73 & 3.55 & 5.31 \\
\hline \multirow[t]{3}{*}{ DMSO } & $\Delta E_{\mathrm{e}}$ & 2.69 & 0.12 & 0.00 & 3.51 & 3.58 & 3.49 & 4.07 & 4.19 & 5.61 & 6.48 & 2.78 & 6.68 \\
\hline & $\Delta H$ & 2.58 & 0.08 & 0.00 & 3.43 & 3.44 & 3.25 & 3.84 & 4.02 & 5.47 & 6.30 & 3.40 & 4.40 \\
\hline & $\Delta G$ & 2.34 & 0.00 & 0.23 & 3.05 & 3.35 & 3.14 & 3.53 & 3.71 & 5.31 & 6.01 & 3.58 & 5.44 \\
\hline \multirow[t]{3}{*}{ water } & $\Delta E_{e}$ & 1.50 & & 0.00 & 2.42 & 2.34 & 2.15 & 2.90 & 3.03 & 3.28 & 4.13 & -2.11 & 6.24 \\
\hline & $\Delta H$ & 1.55 & & 0.00 & 2.62 & 2.40 & 1.81 & 2.63 & 3.12 & 3.36 & 4.16 & 0.02 & 5.11 \\
\hline & $\Delta G$ & 0.74 & & 0.00 & 1.95 & 1.97 & 1.39 & 2.10 & 2.43 & 3.05 & 3.78 & -0.61 & 5.88 \\
\hline
\end{tabular}

"See tootnote $a$ of Table 1 .

of theory. respectively. ${ }^{15}$

At the B3LYP/6-31+G(d) level of theory, the ten local minima are found for N-Ala in the gas phase (Tables 3 and 4). In particular. the two local minima $\mathrm{N} 4$ and $\mathrm{NlO}$ at the $\mathrm{HF} / 6-3 \mathrm{l}+\mathrm{G}(\mathrm{d})$ level of theory do not exist any longer at the $\mathrm{B} 3 \mathrm{LYP} / 6-3 \mathrm{l}+\mathrm{G}(\mathrm{d})$ level of theory. as found at the $\mathrm{B} 3 \mathrm{LYP} / 6-$ $31 \mathrm{l}+\mathrm{G}(\mathrm{d}, \mathrm{p})$ level of theory. ${ }^{1 /}$ The relative conformational stabilities of $\mathrm{N}$-Ala are calculated to be in the order $\mathrm{NI}>$ $\mathrm{N} 2 \mathrm{a}=\mathrm{N} 2 \mathrm{~b}>\mathrm{N} 3>\mathrm{N} 5>\mathrm{N} 6>\mathrm{N} 7>\mathrm{N} 8>\mathrm{N} 9>\mathrm{N} 11$ at the B3LYP/6-31+G(d) level of theory by both the $\Delta E_{\mathrm{e}}$ and $\Delta G$. At the B3LYP/6-311++G(d.p)/B3LYP/6-31+G(d) level of theory. the relative stabilities of local minima are calculated to be similar to those at the B3LYP/6-31+G(d) level of theory. except that the local minimum $\mathrm{N} 2 \mathrm{~b}$ becomes a little more preferred over the local minimum N2a. In particular. the calculated stabilities of local minima at B3LYP/6$31+G(d)$ and B3LYP/6-311++G(d.p)/B3LYP/6-31+G(d) levels of theory are quite similar to those by the $\Delta E_{\mathrm{e}}$ at both the B3LYP/6-3II++G(d,p) and MP2/6-3l $1++G(d, p)$ levels of theory. ${ }^{\text {(i) }}$

At all the levels of theory employed here. the local minimum Nl for N-Ala is found to be most preferred in the gas phase and a weak asymmetric bifurcated hydrogen bond between the amino hydrogens and the carbonyl oxygen appears to play a role in stabilizing this conformation (Figure 2). whose distances are computed to be 2.68 and $2.87 \mathrm{~A}$ at the $\mathrm{HF} / 6-3 \mathrm{l}+\mathrm{G}(\mathrm{d})$ level of theory and 2.68 and $2.94 \mathrm{~A}$ at the B3LYP/6-31+G(d) level of theory. The corresponding values are 2.70 and $2.88 \AA$ at the MP2/6-
$311++\mathrm{G}(\mathrm{d} . \mathrm{p})$ level of theory. ${ }^{7}$ The local minima $\mathrm{N} 2 \mathrm{a}$ and $\mathrm{N} 2 \mathrm{~b}$ are found to be the second preferred conformations at $\mathrm{B} 3 \mathrm{LYP} / 6-3 \mathrm{l}++\mathrm{G}(\mathrm{d} . \mathrm{p}) / \mathrm{HF} / 6-3 \mathrm{l}+\mathrm{G}(\mathrm{d})$. B $3 \mathrm{LYP} / 6-3 \mathrm{l}+\mathrm{G}(\mathrm{d})$, and $B 3 L Y P / 6-3 l l++G(d, p) / B 3 L Y P / 6-3 l+G(d)$ levels of theory. as found at MP2/6-311++G(d.p) ${ }^{7}$ and B3LYP/augcc-pVDZ ${ }^{11}$ levels of theory. The values of $\Delta E_{\mathrm{e}}$ and $\Delta G$ for the local minima $\mathrm{N} 2 \mathrm{a}$ and $\mathrm{N} 2 \mathrm{~b}$ are computed to be 0.36 and $0.95 \mathrm{kcal} / \mathrm{mol}$ and 0.40 and $0.92 \mathrm{kcal} / \mathrm{mol}$, respectively, at the B3LYP/6-311++G(d.p)//HF/6-31+G(d) level of theory: 0.01 and $0.63 \mathrm{kcal} / \mathrm{mol}$ and 0.06 and $0.69 \mathrm{kcal} / \mathrm{mol}$. respectively. at the B3LYP/6-31+G(d) level of theory. 0.03 and $0.66 \mathrm{kcal} / \mathrm{mol}$ and 0.02 and $0.64 \mathrm{kcal} / \mathrm{mol}$, respectively at the B3LYP/6-311++G(d.p)//B3LYP/6-31+G(d) level of theory. These two local minima N2a and N2b seem to be stabilized by a hydrogen bond between the amino nitrogen and the carboxylic hydrogen (Figure 2), with the distances of 2.05 and $2.03 \mathrm{~A}$ at the HF/6-31+G(d) level of theory respectively. and 1.91 and $1.90 \mathrm{~A}$ at the $\mathrm{B} 3 \mathrm{LYP} / 6-31+\mathrm{G}$ (d) level of theory. respectively. The corresponding distance of the local minimum N2a is $1.96 \AA$ at the MP2/6-311++G(d.p) level of theory. ${ }^{7}$ From electron diffraction, ${ }^{6}$ microwave spectroscopy. and matrix-isolation infrared $\mathrm{d}^{1 \mathrm{l}}$ experiments. it has been observed that L-alanine adopts the two most stable conformations NI and N2a. Our results for the relative stabilities obtained at the B3LYP/6-311++G(d.p)//HF/6-31+G(d) and B3LYP/6-31+G(d) levels of theory are reasonably consistent with these observations. although the difference in energy or free energy between two local minima $\mathrm{N} 2 \mathrm{a}$ and $\mathrm{N} 2 \mathrm{~b}$ is not remarkable. 
Although the local minima for Z-Ala and TS are obtained at the HF/6-3I+G(d) level of theory. the free energy of Z-Ala is calculated to be higher than that of TS by 0.65 $\mathrm{kcal} / \mathrm{mol}$ at the $\mathrm{HF} / 6-3 \mathrm{l}+\mathrm{G}(\mathrm{d})$ level of theory and $4.34 \mathrm{kcal} /$ $\mathrm{mol}$ at the B3LYP/6-311++G(d.p)//HF/6-31+G(d) level of theory.

Conformational Preferences in Solution. Thermodynamic properties of N-Ala. Z-Ala. and their TS calculated at HF/6$3 \mathrm{l}+\mathrm{G}(\mathrm{d})$. B3LYP/6-3ll++G(d.p)//HF/6-3l+G(d), B3LYP/ $6-31+\mathrm{G}(\mathrm{d})$. and $\mathrm{B} 3 \mathrm{LYP} / 6-311+\mathrm{G}(\mathrm{d} . \mathrm{p}) / \mathrm{B} 3 \mathrm{LYP} / 6-3 \mathrm{~L}+\mathrm{G}(\mathrm{d})$ levels of theory using the CPCM method in solution are listed in Tables 1-4. respectively. The selected torsion angles of all local minima and TS in solution and their selected bond lengths and bond angles in water at the CPCM HF/6$3 \mathrm{l}+\mathrm{G}(\mathrm{d})$ and $\mathrm{CPCM}$ B $3 \mathrm{LYP} / 6-3 \mathrm{l}+\mathrm{G}(\mathrm{d})$ levels of theory are listed in the Supporting Information. For local minima of N$\mathrm{Ala}$ and Z-Ala and their TS in solution. there are no remarkable shifts in torsion angles, i.e. conformations, from those in the gas phase at both the CPCM HF/6-31+G(d) and CPCM B3LYP/6-31+G(d) levels of theory. At both the CPCM HF and B3LYP levels of theory. most of the local minima for N-Ala and Z-Ala and their TS obtained in the gas phase are retained as stationary points in solution.

At all the levels of theory employed here. the relative stability of the local minimum $\mathrm{N} 2 \mathrm{~b}$ by $\Delta G$ is remarkably increased in solution than that in the gas phase. The local minimum N2b becomes more stable than the local minimum $\mathrm{N} 2 \mathrm{a}$ in most of the solution. In particular. the local minimum N2a does not exist as a stationary point any longer at the CPCM B3LYP $/ 6-31+G(d)$ level of theory in acetonitrile and water.

Although the relative conformational stabilities of N-Ala by $\Delta G$ are different from solution to solution at the $\mathrm{CPCM}$ $\mathrm{HF} / 6-3 \mathrm{I}+\mathrm{G}(\mathrm{d})$ level of theory. the local minimum $\mathrm{Nl}$ is most preferred in most of the solution. except that the local minimum $\mathrm{N} t$ is a little favored than the local minimum N1 in ethanol. However, the local minimum N2b becomes the most preferred conformation by $\Delta G$ and is followed by the local minimum N2a at the B3LYP/6-311++G(d.p)//CPCM $\mathrm{HF} / 6-3 \mathrm{l}+\mathrm{G}(\mathrm{d})$ level of theory in solution. except in ethanol and water. The local minima N4 and N1 are the second preferred conformations in ethanol and water. respectively. which are more stabilized by 0.25 and $0.05 \mathrm{kcal} / \mathrm{mol}$ in $\Delta G$ than the local minimum N2a. respectively. At the CPCM $\mathrm{B} 3 \mathrm{LYP} / 6-31+\mathrm{G}(\mathrm{d})$ and $\mathrm{B} 3 \mathrm{LYP} / 6-311++\mathrm{G}(\mathrm{d} \cdot \mathrm{p}) / \mathrm{CPCM}$ $B 3 L Y P / 6-31+G(d)$ levels of theory in solution. the local minimum N2b appears to be most preferred. although its free energy is higher by 0.08 and $0.27 \mathrm{kcal} / \mathrm{mol}$ than the local minimum N2a in chloroform and DMSO. respectively. In particular. the local minimum N1 is found to be the second preferred conformation in ethanol. acetonitrile and water.

As found in the gas phase. the local minimum N1 for NAla is found to have a weak asy'mmetric bifurcated hydrogen bond between the amino hydrogens and the carbonyl oxygen in solution. whose the shorter distance becomes longer by 0.01-0.02 $\mathrm{A}$ in all the solution and the longer distance are shortened by $0.01-0.03 \AA$ only in ethanol. acetonitrile. and water at the $\mathrm{CPCM} \mathrm{HF} / 6-31+\mathrm{G}(\mathrm{d})$ level of theory. compared to the values at the HF/6-3l+G(d) level of theory in the gas phase. At the CPCM B3LYP/6-31+G(d) level of theory in all the solution. the former distance is a little lengthened by up to $0.03 \AA$ and the latter distance becomes shorter by $0.01 \AA$. except by $0.06 \AA$ in water.

In solution, the two local minima N2b and N2a seem to be stabilized by a hydrogen bond between the amino nitrogen and the carboxylic hydrogen, as seen in the gas phase. At the CPCM HF/6-3l+G(d) level of theory, the hydrogen-bond distances of these two local minima become shorter by 0.03 $0.04 \AA$ in aprotic solutions and a little longer by $0.01 \AA$ in ethanol and water. compared to those in the gas phase. At the CPCM B3LYP/6-3l+G(d) level of theory, these hydrogenbond distances become more shortened by 0.06-0.11 $\AA$ in solution. Thus, the shorter hydrogen-bond distances of these two local minima $\mathrm{N} 2 \mathrm{~b}$ and $\mathrm{N} 2 \mathrm{a}$ seem to play a role in more stabilizing these conformations over the conformation $\mathrm{NI}$ in solution. It should be noted that the local minimum N2a does not exist as a stationary point any longer at this CPCM B3LYP levels of theory in acetonitrile and water as discussed above.

Z-Ala and TS exist as stationary points at both the CPCM $\mathrm{HF} / 6-3 \mathrm{l}+\mathrm{G}(\mathrm{d})$ and $\mathrm{CPCM}$ B $3 \mathrm{LYP} / 6-3 \mathrm{l}+\mathrm{G}$ (d) levels of theory in solution. On the whole the relative free energies of Z-Ala and TS become more lowered at all the levels of theory considered here, as the solvent polarity increases. In particular. Z-Ala becomes more stable than the most preferred conformations $\mathrm{N} 2 \mathrm{~b}, \mathrm{~N} 2 \mathrm{a}$, or NI at all the levels of theory in ethanol and water. except at the B3LYP/6-311++ $\mathrm{G}($ d.p $) / / \mathrm{CPCM}$ B $3 \mathrm{LYP} / 6-3 \mathrm{I}+\mathrm{G}$ (d) level of theory in ethanol.

In water. the relative free energy of Z-Ala to the local minimum Nl is calculated to be $-2.60 \mathrm{kcal} / \mathrm{mol}$ at the CPCM HF/6-3l+G(d) level of theory. which was reported to be $-4.48 \mathrm{kcal} / \mathrm{mol}$ at the $\mathrm{B} 3 \mathrm{LYP} / 6-3 \mathrm{lG}$ (d) level of theory using the polarizable continuum model (PCM) ${ }^{16}$ The relative free energy' of Z-Ala to the local minimum N2b is calculated to be -5.08 and $-3.03 \mathrm{kcal} / \mathrm{mol}$ at B3LYP/6-311++G(d.p)// CPCM HF/6-31+G(d) and CPCM B3LYP/6-31+G(d) levels of theory, respectively. The corresponding relative free energy was computed to be $-3.13 \mathrm{kcal} / \mathrm{mol}$ at the $\mathrm{B} 3 \mathrm{PW} 9 \mathrm{l} / 6$ $31++G(d . p)$ level of theory with the PCM. ${ }^{3 i j}$

Populations of N-Ala and Z-Ala in Solution. The populations of N-Ala and Z-Ala in solution are listed in Table 5. Each population was computed using the normalized Boltzmann weight by $\Delta G$ in solution. shown in Tables 1-4. At the CPCM HF/6-31+G(d). B3LYP/6-311++G(d.p)// CPCM HF $/ 6-31+G(d)$. and CPCM B3LYP/6-31+G(d) levels of theory. N-Ala prevails over Z-Ala in aprotic solutions but Z-Ala is dominantly populated in ethanol and water. In aprotic solutions. the population of $\mathrm{Z}$-Ala increases somewhat with the increase of solvent polarity. except that the population of Z-Ala in DMSO is lower than that in acetonitrile. At the B3LYP/6-311++G(d.p)//CPCM B3LYP/6$31+G$ (d) level of theory. N-Ala prevails over Z-Ala in both aprotic solutions and ethanol. In water. N-Ala is populated 
Table 5. Populations and Ratios of Concentrations for N-Ala and Z-Ala in Solution

\begin{tabular}{|c|c|c|c|c|c|c|c|}
\hline level of theory & pop. & $\mathrm{CHCl}_{2}$ & $\mathrm{CH}_{2} \mathrm{ClCH}_{2} \mathrm{Cl}$ & $\mathrm{EtOH}$ & $\mathrm{CH}_{3} \mathrm{CN}$ & DMSO & water \\
\hline \multirow[t]{3}{*}{$\mathrm{HF} / 6-\hat{3}]+\mathrm{G}(\mathrm{d})$} & $\mathrm{N} \%{ }^{4}$ & 10000 & 10000 & 27.51 & 99.90 & 99.86 & 3.20 \\
\hline & $2 \%$ & 0.00 & 0.00 & 72.49 & 0.10 & 0.14 & 96.80 \\
\hline & $\log K_{z^{c}}$ & -6.09 & -4.43 & 0.42 & $-3,01$ & -2.84 & 1.48 \\
\hline B3LYP/6-311++G(d,p)// & $\mathrm{N} \%{ }^{a}$ & 100,00 & 99.91 & 0.34 & 98.34 & 97.22 & 0.04 \\
\hline \multirow[t]{2}{*}{$\mathrm{HF} / 6-31+\mathrm{G}(\mathrm{d})$} & $\mathrm{Z} \%$ & 0.00 & 0.09 & 99.66 & 1.66 & 2.78 & 99.96 \\
\hline & $\log K_{z}^{c}$ & -4.44 & -3.03 & 2.47 & -1.77 & -1.54 & 3.45 \\
\hline \multirow[t]{3}{*}{ B3LYP/6-31+G(d) } & $\mathrm{N} \%{ }^{a}$ & 99.98 & 99.43 & 4.69 & 91.67 & 95.15 & 0.90 \\
\hline & $Z \%$ & 0.02 & 0.57 & 95.31 & 8.33 & 4.85 & 99.10 \\
\hline & $\log K_{z}^{c}$ & -3.73 & -2.24 & 1.31 & -1.04 & -1.29 & 2.04 \\
\hline B3LYP/6-311++G(d,p)// & $\mathrm{N} \%{ }^{a}$ & 100.00 & 99.98 & 74.11 & 99.76 & 99.86 & 34.83 \\
\hline \multirow[t]{2}{*}{$\mathrm{B} 3 \mathrm{LYP} / 6-31+\mathrm{G}(\mathrm{d})$} & $Z \%$ & 0.00 & 0.02 & 25.89 & 0.24 & 0.14 & 65.17 \\
\hline & $\log K_{z}^{c}$ & -5.20 & -3.78 & -0.46 & -2.63 & -2.86 & 0.27 \\
\hline
\end{tabular}

"Population of N-Ala. "Population of Z-Ala, "The ratio of the concentrations for Z-Ala and N-Ala; $K_{Z}=[Z-A l a]$ [N-Ala $]$.

Table 6. Barriers to Interconversion $\left(\Delta G^{2} Z \rightarrow \Gamma^{-}\right)$of Z-Ala to N-Ala in Solution ${ }^{a}$

\begin{tabular}{lcccrrr}
\hline level of theory & $\mathrm{CHCl}_{3}$ & $\mathrm{CH}_{2} \mathrm{ClCH}_{2} \mathrm{Cl}$ & $\mathrm{EtOH}$ & $\mathrm{CH}_{3} \mathrm{CN}$ & DMSO & water \\
\hline $\mathrm{HF} / 6-31+\mathrm{G}(\mathrm{d})$ & 7.97 & 9.04 & 16.24 & 10.25 & 10.37 & 17.27 \\
$\mathrm{~B} 3 \mathrm{LYP} / 6-31 \mathrm{l}+\mathrm{G}(\mathrm{d}, \mathrm{p}) / \mathrm{HF} / 6-3 \mathrm{l}+\mathrm{G}(\mathrm{d})$ & -0.59 & 0.34 & 7.84 & 1.56 & 1.73 & 8.95 \\
$\mathrm{~B} 3 \mathrm{LYP} / 6-31+\mathrm{G}(\mathrm{d})$ & 1.32 & 2.38 & 7.43 & 3.22 & 3.32 & 8.50 \\
$\mathrm{~B} 3 \mathrm{LYP} / 6-31 \mathrm{l}+\mathrm{G}(\mathrm{d}, \mathrm{p}) / \mathrm{B} 3 \mathrm{LYP} / 6-31+\mathrm{G}(\mathrm{d})$ & 0.15 & 1.03 & 5.46 & 1.76 & 1.86 & 6.49 \\
\hline
\end{tabular}

"Units in kealinol.

by $-35 \%$, although $\mathrm{Z}$-Ala is dominated in water.

The ratios of the concentrations for Z-Ala and N-Ala can be computed using the equation $\log K_{Z}=[\mathrm{Z}-\mathrm{Ala}] /[\mathrm{N}-\mathrm{Ala}]$ and their values are listed in Table 5. The values of $\log K_{Z}$ are positive in ethanol and water at the furst three levels of theory and only in water at the fourth level of theory. Our calculated values of 3.45 and 2.04 for $\log K_{Z}$ at B3LYP/6$3 \mathrm{ll}++\mathrm{G}(\mathrm{d}, \mathrm{p}) / / \mathrm{CPCM} \mathrm{HF} / 6-3 \mathrm{l}+\mathrm{G}(\mathrm{d})$ and CPCM B3LYP/6$3 \mathrm{I}+\mathrm{G}(\mathrm{d})$ levels of theory in water, respectively. are reasonably consistent with the observed value of 5.41 for $\alpha$-alanine in water. ${ }^{3}$ The calculated values of 3.45 and 2.04 for $\log K_{2}$ correspond to 99.96 and $99.10 \%$ of Z-Ala in water, respectively.

Barriers to Z-Ala-to-N-Ala Interconversion in Solution. The barriers to Z-Ala-to-N-Ala interconversion $\left(\Delta G^{\ddagger} Z \rightarrow K^{-}\right)$in solution are shown in Table 6 . The relative free energies of $\mathrm{Z}-\mathrm{Ala}$ and TS to the most preferred conformation of N-Ala at the CPCM B3LYP/6-31+G(d) level of theory in each solution are plotted in Figure 3.

At all the levels of theory, the value of $\Delta G^{\ddagger} \mathrm{Z} \rightarrow \mathrm{N}$ increases on the whole with the increase of solvent polarity. The barriers in ethanol are similar to those in water. although their values in ethanol are lower than those in water. As the solvent polarity increases. the increase in barrier to Z-Ala-to$\mathrm{N}-\mathrm{Ala}$ interconversion is caused by the increase of stability for Z-Ala. as described in the previous section. In particular. the barrier of $-0.59 \mathrm{kcal} / \mathrm{mol}$ at the B3LYP/6-311++G(d.p)// CPCM HF/6-31+G(d) level of theory in chloroform indicates that TS lies $0.59 \mathrm{kcal} / \mathrm{mol}$ below the zwitterionic Z-Ala and the intemediate is not stable. Therefore. the CPCM B3LYP/6-31+G(d) level of theory appears to be the most appropriate method in computing the population and inter-

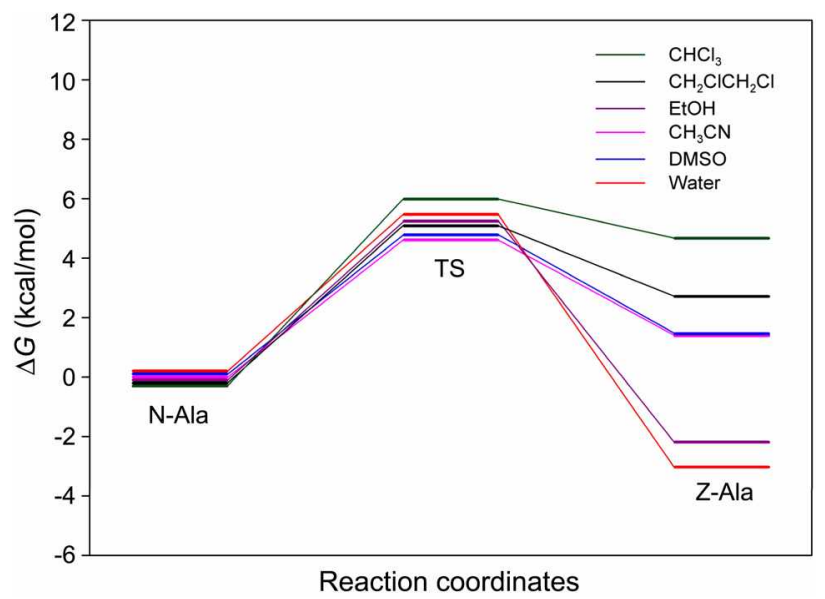

Figure 3. Relative free energies of Z-Ala and TS to the most preferred confonmation of N-Ala at the CPCM BSLYP/6-31+G(d) level of theory in solution.

conversion of neutral and zwitterionic $L$-alanines in solution by the comparison of the ratios of the concentrations for $\mathrm{Z}$ $\mathrm{Ala}$ and $\mathrm{N}-\mathrm{Ala}$ in water and the barrier to Z-Ala-to-N-Ala interconversion in solution.

At the CPCM B3LYP/6-31+G(d) level of theory. the barriers to Z-Ala-to-N-Ala interconversion are computed to be 7.43 .3 .22 . and $8.50 \mathrm{kcal} / \mathrm{mol}$ in ethanol acetonitrile. and water, respectively: These barriers were reported to be 3.38 and $3.85 \mathrm{kcal} / \mathrm{mol}$ at the B3LYP/6-31++G(d.p) level of theory in ethanol and acetonitrile. respectively and 5.86 $\mathrm{kcal} / \mathrm{mol}$ at the $\mathrm{MP} 2 / 6-3 \mathrm{l}+\mathrm{G}(\mathrm{d} . \mathrm{p})$ level of theory in water, ${ }^{-4}$ with the SCRF method using multipolar expansions in an ellipsoidal cavity. 


\section{Conclusions}

At both the HF and B3LYP levels of theory, the local minimum N1 for N-Ala is found to be most preferred in the gas phase and a weak asymmetric bifurcated hydrogen bond between the amino hydrogens and the carbonyl oxygen appears to play a role in stabilizing this conformation. The local minima $\mathrm{N} 2 \mathrm{a}$ and $\mathrm{N} 2 \mathrm{~b}$ are found to be the second preferred confonmations, which seem to be stabilized by a hydrogen bond between the amino nitrogen and the carboxylic hydrogen.

Most of the local minima for N-Ala and Z-Ala and their TS obtained in the gas phase are retained as stationary points at both the CPCM HF and B3LYP levels of theory in solution. The relative stability of the local minimum N2b is remarkably increased in solution than that in the gas phase. The local minimum N2b becomes more stable than the local minimum N2a in most of the solution.

On the whole the relative free energies of Z-Ala and TS become more lowered. as the solvent polarity increases. NAla prevails over Z-Ala in aprotic solutions but Z-Ala is dominantly populated in ethanol and water. In aprotic solutions. the population of $\mathrm{Z}$-Ala increases somewhat with the increase of solvent polarity. The barrier to Z-Ala-to-NAla interconversion increases on the whole with the increase of solvent polarity, which is caused by the increase of stability for Z-Ala. The barriers in ethanol are similar to those in water, although their values in ethanol are lower than those in water.

Acknowledgements. This work is supported by a grant from Chungbuk National University in 2007.

Supporting Information Available. The selected torsion angles of all local minima and transition states in the gas phase and in solution and their selected bond lengths and bond angles in water optimized at the $\mathrm{HF} / 6-3 \mathrm{l}+\mathrm{G}(\mathrm{d})$ and $\mathrm{B} 3 \mathrm{LYP} / 6-3 \mathrm{l}+\mathrm{G}(\mathrm{d})$ levels of theory. This material is avail-

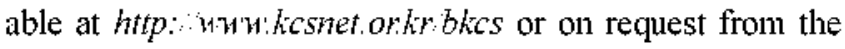
correspondence author.

\section{References}

1. Simpson. H. J.. Jr.: Marsh. R. E. Acta Chst. 1966. 20.550.

2. Lehmann. M. S.: Koetzle. T. F.: Hamilton. W. C. J. Am. Chent. Soc. 1972, 94. 2657.

3. Edsall. J. T.: Blanchard, M. H. J.Am. Chem. Soc. 1933, 55. 2337.

4. Ellzy. M. W.: Jensen, J. O.: Hameka, H. F.: Kay. J. G. Spectrochim. Acta. Part it 2003. 59.2619.

5. Godfrey. P. D.: Firth. S.: Hatherley. L. D.: Brown. R. D.: Pierlot. A. P. J.Amt Chem. Soc. 1993. 115.9687.

6. Iijima, K: Nakano, M. J.M. Mol Struct 1999, 485-486. 255.

7. Blanco, S.: Lesarri. A.; López. J. C.; Alonso. I. L. J. Am. Chem. Soc. 2004, 126.11675.

8. Cao. M: Newton. S. Q.: Pranata. J.: Schäfer. L. THEOCHEMf 1995.332 .251

9. Gronert. S.: O'Hair. R. A. J. J. Am. Chem. Soc. 1995. 117. 2071.

10. Csúszár A. G. J. Phys. Chem. 1996. 100.3541.
11. Stepanian, S. G.: Reva. I. D.: Radchenko, E. D.; Adamowicz. L. J. Phos. Chem. A 1998. 102.4623.

12. Selvarengan. P.: Kolandaivel. P. THEOCHEM 2004. 67]. 77.

13. Kumar. S.; Rai. A. K : Rai. S. B ; Rai, D. K.: Singh, A. N.: Singh. V. B. J. 1 fol. Struct $2006,791.23$

14. Upadhyay, D. M.; Rai, A. K.: Rai. D. K.; Singh, A. N.; Kumar. A. Spectrocitint Acta. Part A 2007. 66.909.

15. Maul. R.: Ortmann. F.: Preuss. M.: Hannewald. K.: Bechstedt. F. J. Contut. Chent. 2007. 28. 1817.

16. Gontrani, L:; Mennucci. B.: Tomasi, J. THEOCHEM 2000. 500. 113 .

17. Frimand, K; Bohr. H; Jalkanen. K. J; Suhai. S. Chem. Phys 2000. 255. 165

18. Jalkanen. K. T.: Nieminen. R. M.: Frimand. K.: Bohr. J.: Bohr. H.: Wade. R. C.: Tajkhorshid. E.: Suhai. S. Chent. Phrs. 2001. 265. 125 .

19. Abdali, S.: Jalkanen. K. J.: Bohr. H.; Suhai, S.; Nieminen. R. M. Chem. Phys. 2002, 282. 219

20. Ahn. D.-S.: Park. S.-W.: Teont. I.-S.: Lee. M.-K.: Kim. N.-H.: Hant. Y.-H.: Lee. S. J. Phis. Chem. B 2003. 107. 14109

21. Chuchev. K.: BelBruno. T. T. THEOCHEM/ 2008. 850.111

22. Cao. X.: Fischer, G. Spectrochim. Acta Part A 1999, 55. 2329.

23. Cao. X.: Fischer. G. Chem. Plys. 2000. 255. 195.

24. Sambrano, J. R; de Sousa, A. R.: Queralt. J. J; Andrés. J.: Longo. E. Chem. Phws. Lett. 1998. 294. I.

25. Nóbrega. G. F.: Sambrano. J. R.: de Souza. A. R.: Queralt. I. J.: Longo. E. THEOCHEM/2001. 5H+151

26. Frisch. M. J.: Trucks. G. W: Scllegel. H. B.: Scuseria, G. E. Robb. M. A.: Cheeseman, J. R.; Montgomery, J. A.. Jr; Vreven. T.: Kudin, K. N.; Burant, J. C.: Millam, J. M. Iyengar. S. S.; Tomasi. J.: Barone. V: Mennucei. B.: Cossi. M.: Scalmani. G.: Rega. N.: Petersson1. G. A.: Nakatsuji. H.: Hada. M.: Ehara. M.: Toyota. K.: Fukuda. R.: Hasegawa. I.: Ishida. M.: Nahajima. T.: Honda, Y; Kitao. O.: Nakai. H.; Klene, M. Li, X; Knox. J. E: Hratchian, H. P; Cross, J. B.; Adamo. C.: Jaramillo. J.: Gomperts. R: Stratmann. R. E.: Yazvev. O: Austin. A. J: Cammi, R: Pomelli. C.: Ochtersk. J. W.: Ayala. P. Y.: Morokuma. K.: Voth. G. A.: Salvador. P.: Dantnenberg. I. T.: Zakizewski. V. G.: Dapprich. S.: Daniels. A. D.: Strain. M. C.: Farkas. O.: Malick. D. K; Rabuck. A. D.: Raghavachari, K; Foresman. J. B; Ortiz, J. V: Cui. Q.: Baboul, A. G.: Clifford. S.: Cioslowski, J.; Stefanov, B. B.: Liu. G. Liashenko, A.; Piskorz, P; Komaromi. I.; Martin. R. L.: Fox. D. J.: Keith. T.: Al-Laham. M. A.: Peng. C. Y: Nanayakikara. A.: Challacombe. M.: Gill. P. M. W.: Tolunson. B.: Chen. W. Wong. M. W: Gonzalez. C.: Pople. T. A. Ganssian 03. revision C.02; Gaussian, Ine.: Wallingford. CT. 2004.

27. Vasquez, M. Nemethy, G. Scheraga. H. A. Macromolecules 1983. 16, 1043

28. Frisch. A.: Denningtonl. R. D.. II: Keith. T. A. Gaussi iew. version 3.0: Gaussian. Inc:- Pittsburgh. PA. 2003.

29. Peng. C.: Schlegel. H. B. Israel J. Chent. 1993. 33. 449

30. Frisch, A.: Frisch. M. J. Trucks. G. W. Gatssian 03 Lser's Reference. version 7.6: Gaussian. Ine.: Pittsburgh, PA, 2003.

31. Barone, V: Cossi, M. J. Phus. Chem \& 1998. 102, 1995

32. Cossi. M.: Rega. N.: Scalmani. G: Barone. V. J. Contut Chen. 2003. 24. 669

33. Barone. V.: Cossi. M.: Tomasi. T. J. Chem. Phus 1997. J07. 3210

34. Cossi, M.; Scalmani. G: Rega. N.: Barone, V. J. Chem Phys. 2002. 117,43

35. Tomasi, J: Mennued. B. Cammi, R. Chem. Rev 2005. 105. 2999

36. Takano. Y: Houk. K. N. J. Chent. The on Contut 2005. 1. 70.

37. Kang. Y. K. THEOCHEM 2001. 546.183.

38. Hehre. W. T.: Radom. L.: Schleyer. P. v. R.: Pople. T. A. At Intio Molecula Orbital Theory: John Wiley \& Sons: New York, 1986: Chapter 6. 